\title{
Confronting fluctuations of conserved charges in central nuclear collisions at the LHC with predictions from Lattice QCD
}

\author{
P. Braun-Munzinger ${ }^{\mathrm{a}, \mathrm{b}, \mathrm{c}}$, A. Kalweit ${ }^{\mathrm{d}, *}$, K. Redlich $^{\mathrm{e}, \mathrm{a}}$, J. Stachel ${ }^{\mathrm{f}}$ \\ a Extreme Matter Institute EMMI, GSI, Planckstr. 1, D-64291 Darmstadt, Germany \\ b Technische Universität Darmstadt, Darmstadt, Germany

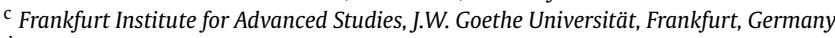 \\ d European Organization for Nuclear Research (CERN), Geneva, Switzerland \\ e Institute of Theoretical Physics, University of Wroclaw, PL-50204 Wrocław, Poland \\ f Physikalisches Institut, Universität Heidelberg, Heidelberg, Germany
}

\section{A R T I C L E I N F O}

\section{Article history:}

Received 2 January 2015

Received in revised form 29 May 2015

Accepted 31 May 2015

Available online 3 June 2015

Editor: J.-P. Blaizot

\begin{abstract}
A B S T R A C T
We construct net baryon number and strangeness susceptibilities as well as correlations between electric charge, strangeness and baryon number from experimental data at midrapidity of the ALICE Collaboration at CERN. The data were taken in central $\mathrm{Pb}-\mathrm{Pb}$ collisions at $\sqrt{s_{N N}}=2.76 \mathrm{TeV}$ and cover one unit of rapidity. The resulting fluctuations and correlations are consistent with Lattice QCD results at the chiral crossover pseudocritical temperature $T_{c} \simeq 155 \mathrm{MeV}$. This agreement lends strong support to the assumption that the fireball created in these collisions is of thermal origin and exhibits characteristic properties expected in QCD at the transition from the quark gluon plasma to the hadronic phase. The volume of the fireball for one unit of rapidity at $T_{c}$ is found to exceed $3000 \mathrm{fm}^{3}$. A detailed discussion on uncertainties in the temperature and volume of the fireball is presented. The results are linked to pion interferometry measurements and predictions from percolation theory.
\end{abstract}

(C) 2015 The Authors. Published by Elsevier B.V. This is an open access article under the CC BY license (http://creativecommons.org/licenses/by/4.0/). Funded by SCOAP ${ }^{3}$.

\section{Introduction}

Uncovering evidence for (partial) restoration of chiral symmetry in the medium created in nucleus-nucleus collisions at very high energy is one of the most important but also challenging problems [1-3]. Recently, experimental studies along this line have been carried out by measuring fluctuations of conserved charges [4-6] as part of the RHIC Beam Energy Scan (BES) program.

Fluctuations of conserved charges are particularly interesting probes of critical phenomena and the phase diagram in QCD [7-11], as well as freezeout conditions in heavy ion collisions [12-15]. The intent of the present work is to provide a link between fluctuations derived from measurements of particle yields in $\mathrm{Pb}-\mathrm{Pb}$ collisions at the $\mathrm{LHC}$ and predictions from Lattice QCD.

Early on, the QCD phase transition was conjectured to be of second order, belonging to the $O(4)$ universality class [16], for massless light quarks. Current Lattice QCD (LQCD) simulations at physical quark masses show that, at vanishing or small baryon density, the transition from a hadron gas to a quark gluon plasma

\footnotetext{
* Corresponding author.

E-mail address: Alexander.Philipp.Kalweit@cern.ch (A. Kalweit).
}

is most likely a crossover [17]. The results further indicate that the chiral crossover appears in the critical region of the second order transition belonging to the $\mathrm{O}(2) / \mathrm{O}(4)$ universality class [18-20]. Consequently, observables such as fluctuations of net baryon number and electric charge, which are sensitive to criticality related with a spontaneous breaking of chiral symmetry, should exhibit characteristic properties governed by the universal part of the free energy $[9,12,21]$.

The magnetic equation of state and cumulants of net charges at physical quark masses have been studied in LQCD calculations [20,22-25], as well as in effective chiral models [21,26-36]. Their properties have been shown to be consistent with general expectations for $O(4)$ scaling. These results have opened a new approach to get experimental information on the QCD phase boundary, by measuring higher moments of distributions of event-by-event fluctuations of conserved charges [12,13,19,21,24,37], and their probability distributions [38-40].

The direct measurement of higher moments of event-by-event fluctuations is complicated by several issues. First, quantities like fluctuations of the net baryon number can only be reliably obtained if effective methods are applied to correct the data for the efficiency of the detector. Furthermore, for conserved quantities 
like baryon number, appropriate corrections need to be applied, due to the finite detector acceptance. The situation has been analyzed by [41,42] and attempts at corrections have been applied for acceptance $[5,6]$ and for fluctuations induced by the difficult to measure neutral baryons $[43,44]$. Second, such measurements are sensitive to critical effects near the QCD phase boundary only for higher moments of the distributions [21], necessitating huge statistics as well as a very precise understanding of possible backgrounds in the measurements. Here we present a different approach, where the second order cumulants and correlations of conserved charges are directly obtained from the measured inclusive distributions, albeit under a special assumption on the shape of the probability distributions.

In the special case that the probability distribution of the number of particles $N_{q}$ and antiparticles $N_{-q}$ is uncorrelated and Poisson, the probability distribution of the variable $N=N_{q}-N_{-q}$ is a Skellam function, which is entirely determined by the mean number of particles $\left\langle N_{q}\right\rangle$ and antiparticles $\left\langle N_{-q}\right\rangle$ [37]. As is explained in the following section one can then determine the second order susceptibilities directly from inclusive measurements.

The assumption of a Skellam distribution for the distribution of net baryon number seems to be well fulfilled at RHIC energies up to the second order cumulants [5,6,37], see also the discussion below. Nevertheless, assuming independent production of baryons and anti-baryons is a strong assumption. However, one should note that generalized susceptibilities $c_{B}^{n}$ do not contain, at least for $\mu_{B}=0$, any singular terms corresponding to chiral critical behavior if $n<6$ [9,21]. Further, there is strong evidence that the fireball is very close to thermal equilibrium as demonstrated by analysis within the framework of the Hadron Resonance Gas (HRG) partition function [45-51], which also quantifies the LQCD equation of state in the confined phase $[24,52,54]$.

The current approach leads then to a direct connection between experimental data integrated over all transverse momenta and second order susceptibilities and, consequently, to direct contact between predictions from LQCD and experimental data without the need to consider, on the experimental side, effects of acceptance and, on the theoretical side, how to extract baryons from LQCD calculations.

\section{Fluctuations and correlations of net charges}

We consider a thermal medium of strongly interacting particles of volume $V$ at temperature $T$, where the baryon number $B$, strangeness $S$ and electric charge $Q$ are conserved on the average. The thermodynamics of such a system is characterized by the pressure, $P(T, V, \vec{\mu})$ in the grand canonical ensemble, where $\vec{\mu}=\left(\mu_{B}, \mu_{S}, \mu_{Q}\right)$ are chemical potentials which guarantee the conservation of all 'charges' $q=(B, Q, S)$.

In this thermal medium, fluctuations of the net charge $N$

$\hat{\chi}_{N} \equiv \frac{\chi_{N}}{T^{2}}=\frac{\partial^{2} \hat{P}}{\partial \hat{\mu}_{N}^{2}}$,

and correlations $\chi_{N, M}$ of charges $N$ and $M$

$\hat{\chi}_{N M} \equiv \frac{\chi_{N M}}{T^{2}}=\frac{\partial^{2} \hat{P}}{\partial \hat{\mu}_{N} \partial \hat{\mu}_{M}}$

are obtained as derivatives of the reduced thermodynamic pressure $\hat{P}=P / T^{4}$, with respect to the corresponding reduced chemical potential $\hat{\mu}_{N}=\mu_{N} / T$, where $N, M=(B, S, Q)$.

The susceptibility of a conserved charge can be also related to its variance,

$\hat{\chi}_{N}=\frac{1}{V T^{3}}\left(\left\langle N^{2}\right\rangle-\langle N\rangle^{2}\right)$.
If $P(N)$ is the probability distribution of a conserved charge $N$, then the $n$-th moment $\left\langle N^{n}\right\rangle$, is calculated as

$\left\langle N^{n}\right\rangle=\sum_{N} N^{n} P(N)$.

For the special case of a Skellam distribution, and from Eqs. (3) and (4), the susceptibility is determined by the total mean number of particles and antiparticles [37],

$\frac{\chi_{N}}{T^{2}}=\frac{1}{V T^{3}}\left(\left\langle N_{q}\right\rangle+\left\langle N_{-q}\right\rangle\right)$

The above result is valid if there are only particles of the same charge, as for baryons, where the charge is $B=1$. For strangeness and an electric charge, there are hadrons with charge two and three. In this case, the Skellam probability distribution can be generalized, and $P(N)$ is expressed by the mean numbers of all particles and antiparticles of different charges $Q, S$ [38]. The net charge susceptibility is then obtained from

$\hat{\chi}_{N}=\frac{\chi_{N}}{T^{2}}=\frac{1}{V T^{3}} \sum_{n=1}^{|q|} n^{2}\left(\left\langle N_{n}\right\rangle+\left\langle N_{-n}\right\rangle\right)$,

where $|q|=(1,2)$ and $|q|=(1,2,3)$ for electric charge and strangeness, respectively.

For the correlation of different charges, the corresponding expression reads

$\hat{\chi}_{N M}=\frac{\chi_{N M}}{T^{2}}=\frac{1}{V T^{3}} \sum_{n=-q_{N}}^{q_{N}} \sum_{m=-q_{M}}^{q_{M}} n m\left\langle N_{n, m}\right\rangle$,

where $\left\langle N_{n, m}\right\rangle$ is the mean number of particles and resonances carrying charges $N=n$ and $M=m$.

\subsection{Modeling susceptibilities and correlations in heavy ion collisions at} the $L H C$

The probability distribution of fluctuations of conserved charges can, in general, be measured in heavy ion collisions using eventby-event analysis. The results for fluctuations of the net baryon, or rather net proton number, obtained by the STAR Collaboration at RHIC $[5,6]$, demonstrate clearly that, in central Au-Au collisions at $\sqrt{S_{N N}}=200 \mathrm{GeV}$, the fluctuations up to third order can be well described by the Skellam distribution. Thus, for small $N$, the distribution $P(N)$ of protons and antiprotons must be independent and very close to Poisson. No dramatic changes in soft particle production have been observed so far when going from RHIC to LHC energy. Consequently, the assumption of independent particle production also at LHC energy seems well founded and, moreover, can be directly tested experimentally.

We take advantage of the above experimental observations, and construct the fluctuations and correlations in central $\mathrm{Pb}-\mathrm{Pb}$ collisions at the LHC by using results of Eqs. (5), (6) and (7). This way we obtain the susceptibilities $\chi_{B}, \chi_{S}$ and $\chi_{Q S}$ from particle yields, measured by the ALICE Collaboration at central rapidity.

The net baryon number fluctuations are obtained as

$$
\begin{aligned}
\frac{\chi_{B}}{T^{2}} & =\frac{1}{V T^{3}}\left[\langle p\rangle+\langle N\rangle+\left\langle\Lambda+\Sigma^{0}\right\rangle+\left\langle\Sigma^{+}\right\rangle+\left\langle\Sigma^{-}\right\rangle\right. \\
& \left.+\left\langle\Xi^{-}\right\rangle+\left\langle\Xi^{0}\right\rangle+\left\langle\Omega^{-}\right\rangle+\text {antiparticles }\right],
\end{aligned}
$$

where \langle\rangle denotes the corresponding mean particle yield per unit rapidity.

The net strangeness susceptibility is calculated following Eq. (6), and approximated as 


$$
\begin{aligned}
\frac{\chi_{S}}{T^{2}} & \simeq \frac{1}{V T^{3}}\left[\left(\left\langle K^{+}\right\rangle+\left\langle K^{0}\right\rangle+\left\langle\Lambda+\Sigma^{0}\right\rangle+\left\langle\Sigma^{+}\right\rangle\right.\right. \\
& \left.+\left\langle\Sigma^{-}\right\rangle+4\left\langle\Xi^{-}\right\rangle+4\left\langle\Xi^{0}\right\rangle+9\left\langle\Omega^{-}\right\rangle+\text {antiparticles }\right) \\
& \left.-\left(\Gamma_{\phi \rightarrow K^{+}}+\Gamma_{\phi \rightarrow K^{-}}+\Gamma_{\phi \rightarrow K^{0}}+\Gamma_{\phi \rightarrow \bar{K}^{0}}\right)\langle\phi\rangle\right] .
\end{aligned}
$$

At LHC energy we assume that $K^{0}=\bar{K}^{0}=K^{+}$and take the experimentally measured value. In the kaon yields $\langle K\rangle$, there are contributions from non-strange resonances decaying into kaons. From Eq. (1), it is clear, that such particles should not contribute to strangeness fluctuations. To correct for the above, we have subtracted kaons coming from $\phi$ decay. The contributions of further non-strange resonances cannot be accounted for since their yields are not known. However, due to their larger masses, such contributions are subleading.

The mixed susceptibilities, from Eq. (2), are selecting contributions of particles carrying the corresponding quantum numbers. We consider, the strangeness-charge correlations $\chi_{Q S}$. Following Eq. (7), the $\chi_{Q S}$ receive contributions only from strange particles with non-vanishing electric charge. We construct strangenesscharge correlations from particle yields as

$$
\begin{aligned}
\frac{\chi Q S}{T^{2}} & \simeq \frac{1}{V T^{3}}\left[\left(\left\langle K^{+}\right\rangle+2\left\langle\Xi^{-}\right\rangle+3\left\langle\Omega^{-}\right\rangle\right.\right. \\
& + \text {antiparticles })-\left(\Gamma_{\phi \rightarrow K^{+}}+\Gamma_{\phi \rightarrow K^{-}}\right)\langle\phi\rangle \\
& \left.-\left(\Gamma_{K_{0}^{*} \rightarrow K^{+}}+\Gamma_{K_{0}^{*} \rightarrow K^{-}}\right)\left\langle K_{0}^{*}\right\rangle\right],
\end{aligned}
$$

where we have again subtracted the contribution from decays of $\phi$ and $K_{0}^{*}$, which are contributing to charged kaon yields, but according to Eq. (2), should not be included. As in the case of $\chi_{S}$, there are also decays of further non-strange, as well as, neutral strange hadrons which are contributing to $\left\langle K^{ \pm}\right\rangle$, but should not be included. However, due to lack of data their contribution cannot be subtracted; nevertheless it is expected to be small.

In heavy ion collisions at the LHC, due to transparency, particles and antiparticles are produced symmetrically at midrapidity. Consequently, the yields of particles and their antiparticle are identical. In addition, at midrapidity, the system is isospin symmetric and charge neutral, thus leading to equal number of protons and neutrons, and, more generally, to equal yields for different charge states of the same particle. Consequently, from Eqs. (8), (9) and (10), one gets

$$
\begin{aligned}
\frac{\chi_{B}}{T^{2}} & =\frac{1}{V T^{3}}\left[4\langle p\rangle+2\left\langle\left(\Lambda+\Sigma^{0}\right)\right\rangle+4\left\langle\Sigma^{+}\right\rangle\right. \\
& +4\langle\Xi\rangle+2\langle\Omega\rangle] \\
\frac{\chi_{S}}{T^{2}} & \simeq \frac{1}{V T^{3}}\left[2\left\langle K^{+}\right\rangle+2\left\langle K^{0}\right\rangle+2\left\langle\left(\Lambda+\Sigma^{0}\right)\right\rangle+4\left\langle\Sigma^{+}\right\rangle\right. \\
& \left.+16\langle\Xi\rangle+18\langle\Omega\rangle-2\left(\Gamma_{\phi \rightarrow K^{+}}+\Gamma_{\phi \rightarrow K^{0}}\right)\langle\phi\rangle\right] \\
\frac{\chi_{Q S}}{T^{2}} & \simeq \frac{1}{V T^{3}}\left[2\left\langle K^{+}\right\rangle+4\left\langle\Xi^{-}\right\rangle+6\left\langle\Omega^{-}\right\rangle-2 \Gamma_{\phi \rightarrow K^{+}}\langle\phi\rangle\right. \\
& \left.-2 \Gamma_{K_{0}^{*} \rightarrow K^{+}}\left\langle K^{*}\right\rangle\right] .
\end{aligned}
$$

\begin{tabular}{|c|c|}
\hline \multicolumn{2}{|c|}{$\begin{array}{l}\text { Table } 1 \\
\text { ALICE data on rapidity distributions at } \\
y=0 \text { for different particle yields in } \\
0-10 \% \text { most central } \mathrm{Pb}-\mathrm{Pb} \text { collisions } \\
\text { at } \sqrt{s}=2.76 \mathrm{TeV}[57-60] \text {. }\end{array}$} \\
\hline$\left\langle\pi^{ \pm}\right\rangle$ & $668.90 \pm 47.50$ \\
\hline$\left\langle K^{+}\right\rangle$ & $99.67 \pm 8.25$ \\
\hline$\left\langle K_{S}^{0}\right\rangle$ & $97.43 \pm 8.00$ \\
\hline$\left\langle K^{*}\right\rangle$ & $19.01 \pm 3.18$ \\
\hline$\langle p\rangle$ & $30.52 \pm 2.50$ \\
\hline$\langle\phi\rangle$ & $12.73 \pm 1.54$ \\
\hline$\left\langle\Lambda+\Sigma^{0}\right\rangle$ & $23.37 \pm 2.50$ \\
\hline$\left\langle\Xi^{-}\right\rangle$ & $3.34 \pm 0.24$ \\
\hline$\left\langle\Omega^{-}\right\rangle$ & $0.60 \pm 0.10$ \\
\hline
\end{tabular}

Furthermore, from data on inclusive $\Lambda$ and $\Sigma^{0}$ production in pBe collisions at $\sqrt{s}=25 \mathrm{GeV}$, we obtain the ratio, $\Sigma^{0} / \Lambda=$ $0.278 \pm 0.011 \pm 0.05$, with a statistical and a systematic error, respectively [55]. We take $\Sigma^{0} / \Lambda=0.278 \pm 0.052$, thus $\langle\Sigma\rangle=$ $(0.2175 \pm 0.032)\left\langle\Lambda+\Sigma^{0}\right\rangle$. The branching ratios, $\Gamma_{\phi \rightarrow K^{0}}=0.342 \pm$ $0.004, \Gamma_{\phi \rightarrow K}=0.489 \pm 0.005$, and $\Gamma_{K_{0}^{*} \rightarrow K^{+}}=0.666$ are from [56].

The charge susceptibilities and correlations between conserved charges can be calculated from the recent ALICE Collaboration data for particle yields per unit rapidity measured in heavy ion collisions at $\sqrt{s}=2.76 \mathrm{TeV}$ at midrapidity, and momentum integrated [57-60]. The results are summarized in Table 1.
The baryon number, strangeness and strangeness-electric charge correlations are obtained from Eq. (11) and from Table 1, as

$$
\begin{aligned}
& \frac{\chi_{B}}{T^{2}}=\frac{1}{V T^{3}}(203.7 \pm 11.44) \\
& \frac{\chi_{S}}{T^{2}} \simeq \frac{1}{V T^{3}}(504.35 \pm 24.14) \\
& \frac{\chi_{Q S}}{T^{2}} \simeq \frac{1}{V T^{3}}(178.5 \pm 17.14) .
\end{aligned}
$$

Particularly interesting are the susceptibility ratios,

$$
\frac{\chi_{B}}{\chi_{S}} \simeq 0.404 \pm 0.028, \frac{\chi_{B}}{\chi_{Q S}} \simeq 1.141 \pm 0.1266
$$

which are independent of temperature and volume.

In Eqs. (12) to (15) the uncertainties of rapidity densities for particles and their antiparticles (apart from absorption corrections) were assumed to be fully correlated and therefore were added linearly. All remaining errors were treated as being independent, thus were added in quadrature. In the calculation of the errors of different susceptibility ratios, the partial cancelation of errors due to particles which appear both in the nominator and denominator has been explicitly taken into account.

\subsection{Relating $L H C$ data to $L Q C D$}

The net baryon number and strangeness susceptibilities, as well as the electric charge-strangeness correlations, have been recently calculated in LQCD at $\mu_{B}=0$ for different temperatures [20, $22-25]$. The results are extrapolated to the continuum limit, thus can be directly compared to heavy ion data.

One expects that a fireball created in heavy ion collisions is of thermal origin and its properties are governed by statistical QCD, as quantified by LQCD. If there is a phase change from QGP to the hadronic phase, then the particle yields and fluctuations of conserved charges should be established at the chiral, pseudocritical temperature $T_{c}$. The value of $T_{c}$ is well established by LQCD and coincides within a different discretization scheme of fermionic action. The value, $T_{c}=155$ (1)(8) was recently obtained in LQCD with domain wall fermions [61], which preserves all relevant symmetries of QCD.

The most transparent way to check if the fluctuations of conserved charges, extracted from ALICE data, are consistent with LQCD at $T \simeq T_{c}$, is to compare the ratios from Eq. (15) to the corresponding LQCD results.

In Fig. 1, we compare $\chi_{B} / \chi_{S}, \chi_{B} / \chi_{Q S}$ and $\chi_{S} / \chi_{Q S}$ ratios with the continuum limit extrapolated LQCD values at pseudocritical temperature, $T_{c}=155 \mathrm{MeV}$ [24,25]. Fig. 1 shows that, within systematic uncertainties, there is a very good agreement between $\mathrm{Pb}-\mathrm{Pb}$ collision data from the ALICE experiment at the LHC and the LQCD results at $T \simeq 155 \mathrm{MeV}$. 


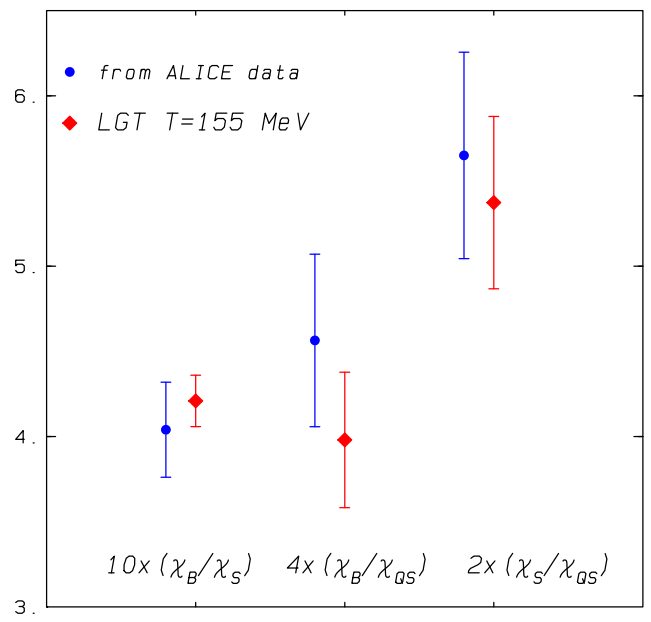

Fig. 1. Comparison of different susceptibility ratios obtained by using data measured by the ALICE Collaboration in Eq. (15) with LQCD results at $T=155 \mathrm{MeV}$ from Refs. [24,25].

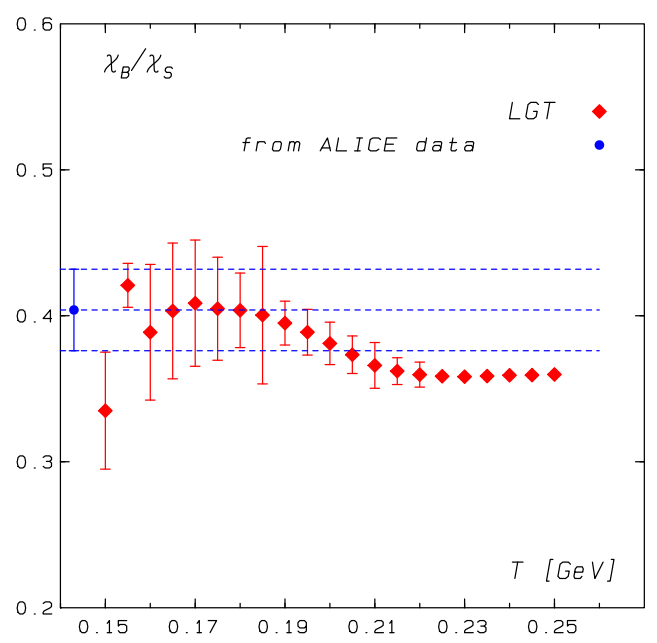

Fig. 2. The LQCD results on temperature dependent baryon number and strangeness susceptibility ratio from Ref. [24]. The LQCD value at $T=155 \mathrm{MeV}$ is from Ref. [25]. Also shown is a band for the expected value of this ratio constructed from ALICE data in Eq. (15).

However, the value of the temperature, at which experimental results and theory predictions agree, cannot be uniquely determined by comparing ratios shown in Fig. 1. This is illustrated in Figs. 2 and 3 , where experimental results for $\chi_{B} / \chi_{S}$ and $\chi_{B} / \chi_{Q S}$ from Eq. (15) are compared with LGCD predictions at different temperatures [25].

The LQCD susceptibility ratios exhibit a rather weak temperature dependence, and for $T>0.15 \mathrm{GeV}$, are consistent, within statistical and systematic uncertainties, with results obtained by using ALICE data. From Figs. 2 and 3, one can exclude temperatures $T \leq 0.15 \mathrm{GeV}$ as a possible range where the saturation of fluctuations in heavy ion data appears. The upper limit, on the other hand, can be as large as $0.21 \mathrm{GeV}$.

However, based on different combinations of charge fluctuations and correlations, it was shown, that at $T>163 \mathrm{MeV}$, the LQCD thermodynamics cannot be anymore described by hadronic degrees of freedom [54]. This argument reduces a conceivable window for the saturation of the net baryon number and strangeness fluctuations to $0.15<T<0.163 \mathrm{GeV}$.

Further constraints on the lower temperature limit for chemical freezeout in heavy ion collisions at the LHC can be also obtained

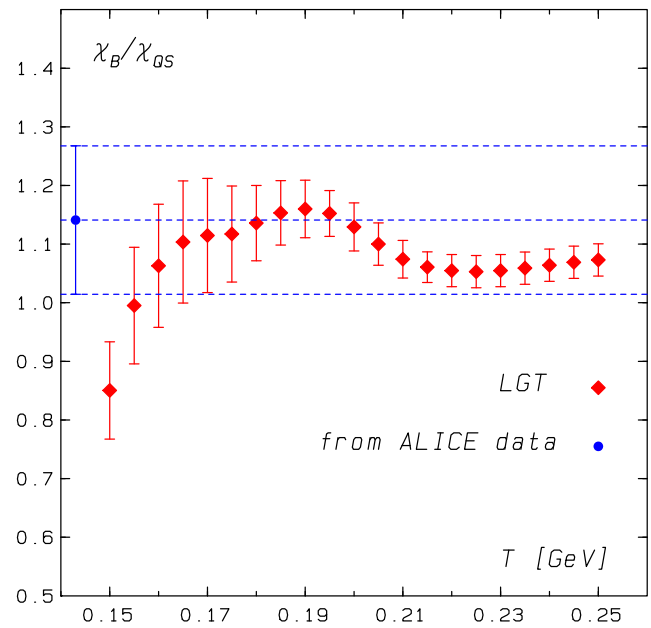

Fig. 3. Ratio $\chi_{B} / \chi_{Q S}$ from LQCD data from Refs. [24,25], and obtained from ALICE data in Eq. (15).

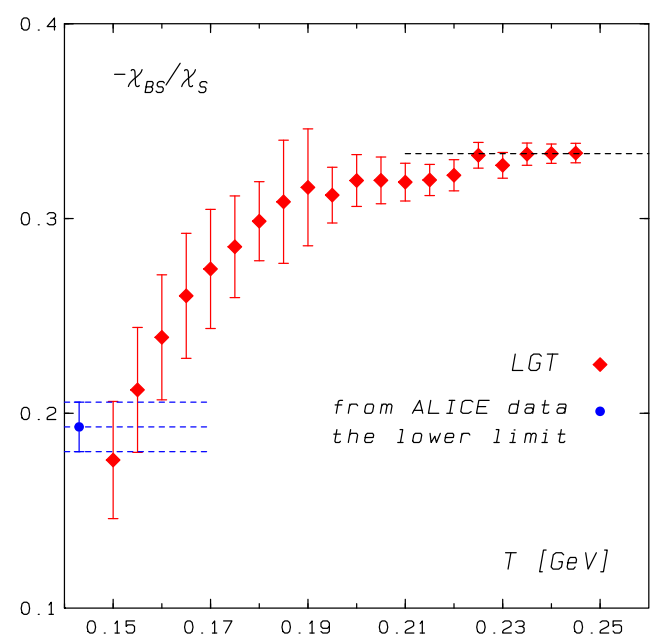

Fig. 4. The LQCD results on temperature dependent ratio of baryon-strangeness correlation $\chi_{B S}$ and strangeness susceptibility from Ref. [24]. Also shown is a band for the lower limit on this ratio extracted from ALICE data from Eqs. (13) and (16). The horizontal line at high- $T$ is an ideal gas value in a QGP.

by considering correlations between strangeness and baryon number, $\chi_{B S}$. Particulary interesting is the ratio $\chi_{B S} / \chi_{S}$, which was proposed as a diagnostic observable for deconfinement [53].

The $\chi_{B S}$ correlations are obtained from Eq. (7). Their upper limit can be expressed by yields of measured strange baryons by ALICE Collaboration, as

$$
\begin{aligned}
-\frac{\chi_{B S}}{T^{2}} & >\frac{1}{V T^{3}}\left[2\left\langle\Lambda+\Sigma^{0}\right\rangle+4\left\langle\Sigma^{+}\right\rangle\right. \\
& \left.+8\langle\Xi\rangle+6\left\langle\Omega^{-}\right\rangle\right]=97.4 \pm 5.8 .
\end{aligned}
$$

Eq. (16) sets only an upper limit for $\chi_{B S}$ since, e.g., the contributions of strange baryonic resonances decaying into non-strange baryon and strange meson, like decay of $\Sigma^{*} \rightarrow N \bar{K}$, are not included as they are not known experimentally.

In Fig. 4 we show the $\left(-\chi_{B S} / \chi_{S}\right)$ ratio obtained in LQCD by the HotQCD Collaboration [24]. The LQCD results are compared with the lower limit, $\left(-\chi_{B S} / \chi_{S}\right)>0.193 \pm 0.0127$, obtained from Eqs. (13) and (16), and ALICE data summarized in Table 1. A strong increase of this ratio with temperature, makes it an ideal observable to fix the temperature in HIC through a direct comparison of data to LQCD results. From Fig. 4, it is clear that data are pointing 


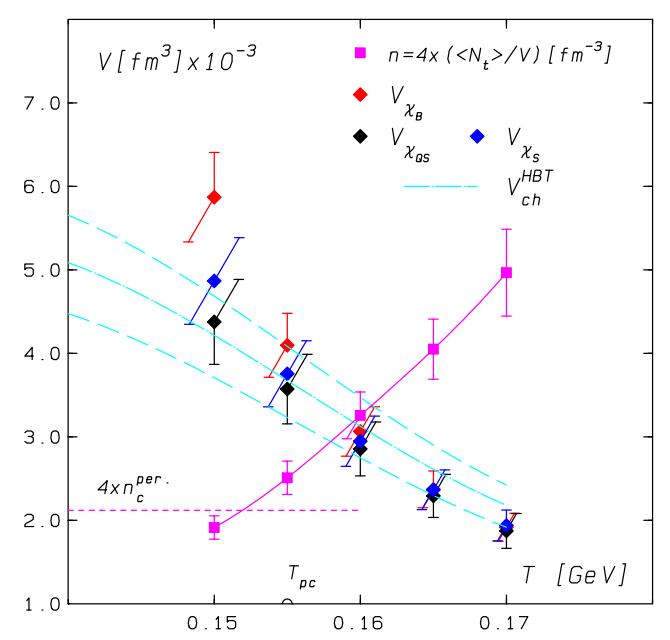

Fig. 5. Volume calculated from Eqs. (17) and (18). Also shown are results for particle density at corresponding temperature and the critical density in percolation theory, as well as the chemical freezeout volume $V_{c h}^{H B T}$, extracted from the HBT data at thermal freezeout and rescaled to higher $T$ within $3 \mathrm{D}$-hydrodynamics.

towards temperatures $T>0.15 \mathrm{GeV}$. This supports the conclusion already drawn from Figs. 2 and 3.

The agreement of the fluctuation ratios extracted from ALICE data and LQCD in the chiral crossover, seen in Figs. 1-4, supports our assumption, that at the QCD phase boundary, the second order cumulants and charge correlations are well approximated by an uncorrelated particle production.

The susceptibilities in Eqs. (12), (13) and (14) can also be used to obtain information on the volume of the fireball for one unit of rapidity,

$V_{\chi_{B}}=\frac{203.7 \pm 11.44}{T^{3}\left(\chi_{B} / T^{2}\right)_{L Q C D}}, \quad V_{\chi_{S}}=\frac{504.35 \pm 24.14}{T^{3}\left(\chi_{S} / T^{2}\right)_{L Q C D}}$

and

$V_{\chi_{Q S}}=\frac{178.5 \pm 17.14}{T^{3}\left(\chi_{Q S} / T^{2}\right)_{L Q C D}}$.

Clearly, if $\chi_{B}, \chi_{S}$ and $\chi_{Q S}$ are established in a common fireball, then not only the temperature, but also the corresponding volumes, $V_{\chi_{B}}, V_{\chi_{S}}$ and $V_{\chi_{Q S}}$ must be equal.

In Fig. 5, we show the temperature dependence of volume parameters obtained from Eqs. (17) and (18). There is a clear decrease of volume with temperature, which is needed to reproduce LQCD susceptibilities. For a given temperature, the volume of the fireball is extracted as overlap of all $V_{\chi_{B}}, V_{\chi_{S}}$ and $V_{\chi_{Q S}}$.

The volume parameters from Fig. 5 , together with the total number of particles in the final state $\left\langle N_{t}\right\rangle$, are used to calculate the density of particles in a collision fireball, $n(T)=\left\langle N_{t}\right\rangle / V(T)$.

We calculate the total number of particles per unit of rapidity at midrapidity in central $\mathrm{Pb}-\mathrm{Pb}$ collisions at $\sqrt{s}=2.76 \mathrm{TeV}$, as follows

$$
\begin{aligned}
\left\langle N_{t}\right\rangle & =3\langle\pi\rangle+4\langle K\rangle+4\langle p\rangle+2\left\langle\Lambda+\Sigma^{0}\right\rangle+4\langle\Sigma\rangle \\
& +4\langle\bar{\Xi}\rangle+2\langle\bar{\Omega}\rangle,
\end{aligned}
$$

which gives $\left\langle N_{t}\right\rangle=2486 \pm 146$

In Fig. 5, we show the corresponding density of particles, $n(T)$. Clearly, due to deconfinement, there is a limiting temperature and corresponding density, above which the fireball constituents cannot be hadronic anymore.

In percolation theory of objects of (eigen-)volume $V_{0}$, there is a critical density, $n_{c}^{\text {per }}=1.22 / V_{0}$ beyond which the objects start to overlap [62]. Relating percolation to deconfinement [62], one can estimate the critical particle density in the hadronic phase. Considering hadrons as objects of volume $V_{0}=(4 / 3) \pi R_{0}^{3}$, with $R_{0}=\sqrt{\left\langle r_{p}^{2}\right\rangle}$ and $\left\langle r_{p}^{2}\right\rangle=0.67 \pm 0.02$ being the mean squared strong interaction radius of the proton [63], ${ }^{1}$ one gets, $n_{c}^{\text {per }} \simeq 0.53 \pm$ $0.024 \mathrm{fm}^{-3}$. The central $n_{c}^{\text {per }}$ value is also marked in Fig. 4 .

Remarkably, this critical percolation density appears at $T=$ $152 \pm 1 \mathrm{MeV}$, thus within systematic uncertainties, is consistent with the transition temperature obtained from LQCD. Clearly, the value of $T$ at $n_{c}^{\text {per }}$ strongly depends on $R_{0}$. The lower limit of $R_{0} \simeq 0.67 \mathrm{fm}$ corresponds to $T \simeq 163 \mathrm{MeV}$, since above this temperature, the LQCD thermodynamics is not anymore described by the hadronic degrees of freedom. This lower value of $R_{0}$ coincides, within error, with a measured charge radius of the pion, $\sqrt{\left\langle r_{\pi}^{2}\right\rangle}=0.657 \pm 0.012$ [65]. Consequently, the percolation of pions and protons appears at temperatures which overlap with the QCD chiral crossover.

Some limitations on the volume of the fireball, thus also on temperature, can be imposed from the HBT interferometry measurements. The Hanbury-Brown-Twiss (HBT) analysis of multiparticle production processes is becoming a widely used technique in heavy ion collisions. It provides information on the space-time evolution of an excited strongly interacting system produced in high energy collisions.

The HBT volume, can be obtained from the product of the longitudinal $R_{l}$, outward $R_{O}$ and the sideward $R_{S}$ radius, as $V_{H B T}=$ $(2 \pi)^{3 / 2} R_{l} R_{0} R_{s}$, if the $R_{i}$ are rms values of Gaussian distributions. From the first measurement of two-pion Bose-Einstein correlations in central $\mathrm{Pb}-\mathrm{Pb}$ collisions at $\sqrt{s_{N N}}=2.76 \mathrm{TeV}$ at the LHC by ALICE Collaboration [66], one gets $V_{H B T}=4800 \pm 580 \mathrm{fm}^{3}$ for centrality $(0-5 \%)$. We note, however, that $V_{H B T}$ is, in general, considered as the volume at thermal freeze-out. Thus, the fireball volume at chemical freezeout $V_{c h}^{H B T}(T)$ is smaller than the $V_{H B T}$ introduced above due to the expansion of the system between chemical and thermal freeze-out. To connect $V_{C H}^{H B T}(T)$ with $V_{H B T}$ involves model assumptions which we discuss briefly below.

Furthermore, $V_{H B T}$ is not representing the source size, but only the volume of the homogeneity region at the last interaction. Following the procedure developed in [67] we estimate that the true thermal freeze-out volume per unit of rapidity exceeds the $V_{H B T}$ value above by a factor of 1.28 for thermal freeze-out at $T=$ $155 \mathrm{MeV}$, by 1.47 at $T=120 \mathrm{MeV}$ and by 1.63 at $T=100 \mathrm{MeV}$. We further note that $V_{H B T}$ grows with the charge particle multiplicity [66], as expected from the fireball volume at chemical freeze out. As a consequence we use the volume appropriate for (0-10\%) centrality and correct with the above factors.

The relevant corrected volumes are then $V_{c h}=5510 \pm 670 \mathrm{fm}^{3}$ for $T=155 \mathrm{MeV}, V_{c h}=6340 \pm 770 \mathrm{fm}^{3}$ for $T=120 \mathrm{MeV}$ and $V_{c h}=7050 \pm 850 \mathrm{fm}^{3}$ at $T=100 \mathrm{MeV}$. For thermal freeze-out at $155 \mathrm{MeV}$, i.e. close to the chiral crossover temperature, there is no further extrapolation needed, and the minimal corrected volume is $V_{c h}^{H B T}=4840 \mathrm{fm}^{3}$. As seen in Fig. 5, this chemical freezeout volume is within uncertainies comparable with that extracted from the LQCD analysis at temperature $T=155 \mathrm{MeV}$.

For thermal freeze-out at $T=100$ and $T=120 \mathrm{MeV}$, one can calculate the volume decrease with increasing temperature, i.e. towards the chiral crossover temperature, by employing models for expansion dynamics in heavy ion collisions. We have here adopted the 3D-hydrodynamics approach with initial conditions appropriate for LHC energy and calculated in the MC Glauber model to

\footnotetext{
1 This hadronic radius of the proton is somewhat smaller than its recently obtained charge radius, $r_{p}^{E}=0.84 \pm 0.01$ [64].
} 
extract the relative change of the fireball volume with temperature [68].

In Fig. 5 we show the $T$-dependent fireball volume $V_{c h}^{H B T}(T)$ obtained by rescaling the above kinetic freezeout volume at $T=$ $100 \mathrm{MeV}$ with the factor obtained from the 3D-hydrodynamics for a temperature range between 140 and $170 \mathrm{MeV}$. Starting from the kinetic freezeout volume at $T=120 \mathrm{MeV}$ leads to very similar results.

As seen in Fig. 5, the $V_{c h}^{H B T}(T)$ coincides with the volume extracted from LQCD and ALICE data, in the chiral crossover region. This by itself is a non-trivial observation. At the same time, such comparison does not restrict the value of the chemical freezeout temperature at LHC energy.

From the comparison of different fluctuation ratios extracted from ALICE data and LQCD results, one concludes that, in heavy ion collisions at the LHC, the second order fluctuations are of thermal origin and saturate at LQCD values at temperature $T>0.15 \mathrm{GeV}$. This together with the LQCD observation, that at $T>0.163 \mathrm{GeV}$, the fluctuations of conserved charges cannot be anymore described by the hadronic degrees of freedom implies that $0.15<T_{c}<0.163$ is the most likely temperature range for particle freezout at the LHC. In this temperature window, which overlaps with the chiral crossover temperature, the $T$-correlated fireball volume per unit rapidity is obtained from Fig. 5 as $5000>V \geq 3000 \mathrm{fm}^{3}$. This range of volumes is also consistent with that extracted from the HBT measurement and extrapolated to higher temperatures within 3D-hydrodynamics [68].

A recent analysis of particle yields in heavy ion collisions at the LHC, within the thermal model, has shown that $T \simeq 156 \mathrm{MeV}$ and $V \simeq 5300 \mathrm{fm}^{3}$, reproduce all yield data [50]. This temperature value agrees well with the present analysis. The somewhat larger volume in Ref. [50] appears, since repulsive interactions of particles were included in the analysis. In this case, particle densities are reduced, and to reproduce measured yields, a larger volume is required.

\section{Concluding remarks}

We have proposed a method to construct the net baryon number and strangeness susceptibilities as well as correlations between electric charge and strangeness from experimental data of the ALICE Collaboration, taken in Pb-Pb collisions at $\sqrt{S_{N N}}=2.76 \mathrm{TeV}$.

Using this approach, we have shown that fluctuations and correlations derived from ALICE data at the LHC are consistent with LQCD predictions in the temperature window, $0.15<T_{c} \leq$ $0.163 \mathrm{GeV}$, which overlap with the chiral crossover. In this temperature interval, the fireball volume per unit rapidity corresponds to $5000>V \geq 3000 \mathrm{fm}^{3}$.

Such a direct agreement between experiment and LQCD lends strong support to the notion that the fireball created in central nucleus-nucleus collisions at the LHC is of thermal origin and exhibits characteristic properties expected in QCD at the transition from a quark-gluon plasma to a hadronic phase.

We have discussed uncertainties in the determination of temperature and volume of the fireball at the LHC. We have also discussed possible constraints on the parameters originating from pion interferometry measurements and percolation theory.

The analysis presented here provides the first direct link between LHC heavy ion data and predictions from LQCD. This was possible since, at the LHC, the conditions of charge neutrality in the fireball directly match that in LQCD calculations. In addition, the constructed susceptibilities and correlations contain contributions from all charged hadrons integrated over the full momentum range. This is essential and necessary to make a successful comparison of data to the first principle LQCD calculations.
Finally, our method is based on the assumption that the probability distribution of baryons at LHC energy is close to a Skellam distribution. The probability distribution for net baryon number production at LHC energy can be directly obtained from measurements of protons and antiprotons. Since we are interested in results at midrapidity, issues related to isospin can be safely neglected and proton and neutron mean numbers should be equal. Furthermore, corrections due to baryon number conservation should be negligible since near midrapidity the baryon rapidity distribution is very close to flat. Since the present method only relies on the second moment of the distribution, very high statistics is not needed and a typical $10^{6}$ central collisions should be sufficient, implying that our assumption can be tested experimentally in the near future.

\section{Acknowledgements}

We acknowledge stimulating discussions with Bengt Friman and Frithjof Karsch. We also acknowledge fruitful critical comments from Volker Koch. We are grateful to Vladimir Shapoval and Yuri Sinyukov for fruitful discussions and for providing results from hydrodynamics calculations. This work was partly supported by the Polish Science Foundation (NCN), under Maestro grant DEC-2013/10/A/ST2/00106.

\section{References}

[1] B. Friman, C. Höhne, J. Knoll, S. Leupold, J. Randrup, R. Rapp, P. Senger, Lect. Notes Phys. 814 (2011) 1

[2] K. Fukushima, T. Hatsuda, Rep. Prog. Phys. 74 (2011) 014001

[3] K. Fukushima, C. Sasaki, Prog. Part. Nucl. Phys. 72 (2013) 99.

[4] M.M. Aggarwal, et al., STAR Collaboration, Phys. Rev. Lett. 105 (2010) 022302

[5] L. Adamczyk, et al., STAR Collaboration, Phys. Rev. Lett. 112 (2014) 032302.

[6] L. Adamczyk, et al., STAR Collaboration, Phys. Rev. Lett. 113 (2014) 092301.

[7] Y. Hatta, M.A. Stephanov, Phys. Rev. Lett. 91 (2003) 102003

[8] M. Stephanov, K. Rajagopal, E. Shuryak, Phys. Rev. Lett. 81 (1998) 4816

[9] S. Ejiri, F. Karsch, K. Redlich, Phys. Lett. B 633 (2006) 275.

[10] M.A. Stephanov, Phys. Rev. Lett. 102 (2009) 032301.

[11] M.A. Stephanov, Phys. Rev. Lett. 107 (2011) 052301.

[12] F. Karsch, K. Redlich, Phys. Lett. B 695 (2011) 136.

[13] A. Bazavov, H.T. Ding, P. Hegde, O. Kaczmarek, F. Karsch, E. Laermann, S. Mukherjee, P. Petreczky, Phys. Rev. Lett. 109 (2012) 192302.

[14] C. Ratti, et al., Wuppertal-Budapest Collaboration, Nucl. Phys. A 855 (2011) 253.

[15] S. Borsanyi, Z. Fodor, S.D. Katz, S. Krieg, C. Ratti, K.K. Szabo, Phys. Rev. Lett. 113 (2014) 052301

[16] R.D. Pisarski, F. Wilczek, Phys. Rev. D 29 (1984) 338.

[17] Y. Aoki, G. Endrödi, Z. Fodor, S.D. Katz, K.K. Szabó, Nature 443 (2006) 675

[18] S. Ejiri, F. Karsch, E. Laermann, C. Miao, S. Mukherjee, P. Petreczky, C. Schmidt, W. Soeldner, W. Unger, Phys. Rev. D 80 (2009) 094505.

[19] O. Kaczmarek, F. Karsch, E. Laermann, C. Miao, S. Mukherjee, P. Petreczky, C. Schmidt, W. Soeldner, W. Unger, Phys. Rev. D 83 (2011) 014504.

[20] C.R. Allton, M. Döring, S. Ejiri, S.J. Hands, O. Kaczmarek, F. Karsch, E. Laermann, K. Redlich, Phys. Rev. D 71 (2005) 054508

[21] B. Friman, F. Karsch, K. Redlich, V. Skokov, Eur. Phys. J. C 71 (2011) 1694.

[22] S. Borsanyi, Z. Fodor, S.D. Katz, S. Krieg, C. Ratti, K. Szabo, Wuppertal-Budapest Collaboration, J. High Energy Phys. 1201 (2012) 138.

[23] M. Cheng, P. Hedge, C. Jung, F. Karsch, O. Kaczmarek, E. Laermann, R.D. Nawhinney, C. Miao, P. Petreczky, C. Schmidt, Phys. Rev. D 79 (2009) 074505.

[24] A. Bazavov, et al., HotQCD Collaboration, Phys. Rev. D 86 (2012) 034509.

[25] A. Bazavov, H.-T. Ding, P. Hegde, O. Kaczmarek, F. Karsch, E. Laermann, Y. Maezawa, S. Mukherjee, Phys. Rev. Lett. 113 (2014) 072001.

[26] K. Fukushima, Phys. Lett. B 591 (2004) 277.

[27] C. Sasaki, B. Friman, K. Redlich, Phys. Rev. D 75 (2007) 054026.

[28] C. Sasaki, B. Friman, K. Redlich, Phys. Rev. D 75 (2007) 074013

[29] B. Stokic, B. Friman, K. Redlich, Phys. Lett. B 673 (2009) 192.

[30] V. Skokov, B. Stokic, B. Friman, K. Redlich, Phys. Rev. C 82 (2010) 015206

[31] V. Skokov, B. Friman, E. Nakano, K. Redlich, B.-J. Schaefer, Phys. Rev. D 82 (2010) 034029.

[32] V. Skokov, B. Friman, K. Redlich, Phys. Rev. C 83 (2011) 054904.

[33] M. Asakawa, S. Ejiri, M. Kitazawa, Phys. Rev. Lett. 103 (2009) 262301.

[34] T.K. Herbst, J.M. Pawlowski, B.J. Schaefer, Phys. Lett. B 696 (2011) 58.

[35] B.J. Schaefer, M. Wagner, Phys. Rev. D 85 (2012) 034027.

[36] M. Wagner, A. Walther, B.J. Schaefer, Comput. Phys. Commun. 181 (2010) 756 
[37] P. Braun-Munzinger, B. Friman, F. Karsch, K. Redlich, V. Skokov, Phys. Rev. C 84 (2011) 064911.

[38] P. Braun-Munzinger, B. Friman, F. Karsch, K. Redlich, V. Skokov, Nucl. Phys. A 880 (2012) 88.

[39] K. Morita, V. Skokov, B. Friman, K. Redlich, Eur. Phys. J. C 74 (2014) 2706.

[40] K. Morita, B. Friman, K. Redlich, V. Skokov, Phys. Rev. C 88 (2013) 034903, arXiv:1301.2873.

[41] A. Bzdak, V. Koch, Phys. Rev. C 86 (2012) 044904.

[42] A. Bzdak, V. Koch, V. Skokov, Phys. Rev. C 87 (2013) 014901.

[43] M. Kitazawa, M. Asakawa, Phys. Rev. C 85 (2012) 021901.

[44] M. Kitazawa, M. Asakawa, Phys. Rev. C 86 (2012) 024904.

[45] P. Braun-Munzinger, I. Heppe, J. Stachel, Phys. Lett. B 465 (1999) 15.

[46] P. Braun-Munzinger, D. Magestro, K. Redlich, J. Stachel, Phys. Lett. B 518 (2001) 41.

[47] P. Braun-Munzinger, K. Redlich, J. Stachel, in: R.C. Hwa, X.N. Wang (Eds.), Quark-Gluon Plasma 3, World Scientific, 2004.

[48] A. Andronic, P. Braun-Munzinger, K. Redlich, J. Stachel, J. Phys. G 38 (2011) 124081.

[49] A. Andronic, P. Braun-Munzinger, K. Redlich, J. Stachel, Nucl. Phys. A 904-905 (2013) 535c

[50] J. Stachel, A. Andronic, P. Braun-Munzinger, K. Redlich, J. Phys. Conf. Ser. 509 (2014) 012019.
[51] F. Becattini, E. Grossi, M. Bleicher, J. Steinheimer, R. Stock, Phys. Rev. C 90 (2014) 054907.

[52] F. Karsch, K. Redlich, A. Tawfik, Phys. Lett. B 571 (2003) 67; F. Karsch, K. Redlich, A. Tawfik, Eur. Phys. J. C 29 (2003) 549.

[53] V. Koch, A. Majumder, J. Randrup, Phys. Rev. Lett. 95 (2005) 182301.

[54] F. Karsch, Acta Phys. Polon. Supp. 7 (1) (2014) 117.

[55] Marilyn W. Sullivan, et al., Phys. Rev. D 36 (1978) 674.

[56] K.A. Olive, et al., Particle Data Group, Chin. Phys. C 38 (2014) 090001.

[57] B. Abelev, et al., ALICE Collaboration, Phys. Rev. C 88 (4) (2013) 044910

[58] B.B. Abelev, et al., ALICE Collaboration, Phys. Lett. B 728 (2014) 216;

B.B. Abelev, et al., ALICE Collaboration, Phys. Lett. B 734 (2014) 409 (Erratum).

[59] B.B. Abelev, et al., ALICE Collaboration, Phys. Rev. Lett. 111 (22) (2013) 222301.

[60] B.B. Abelev, et al., ALICE Collaboration, Phys. Rev. C 91 (2015) 02409.

[61] T. Bhattacharya, M.I. Buchoff, N.H. Christ, H.-T. Ding, R. Gupta, C. Jung, F. Karsch, Z. Lin, Phys. Rev. Lett. 113 (2014) 082001.

[62] P. Castorina, K. Redlich, H. Satz, Eur. Phys. J. C 59 (2009) 67.

[63] P. Povh, J. Hüfner, Phys. Lett. B 245 (1990) 653.

[64] I.T. Lorenz, H.-W. Hammer, Ulf-G. Meissner, Eur. Phys. J. A 48 (2012) 151.

[65] S.R. Amendolia, et al., Phys. Lett. B 146 (1984) 116.

[66] K. Aamodt, et al., ALICE Collaboration, Phys. Lett. B 696 (2011) 328.

[67] D. Adamova, et al., CERES Collaboration, Phys. Rev. Lett. 90 (2003) 022301.

[68] S.V. Akkelin, P. Braun-Munzinger, Y.M. Sinyukov, Nucl. Phys. A 710 (2002) 439. 